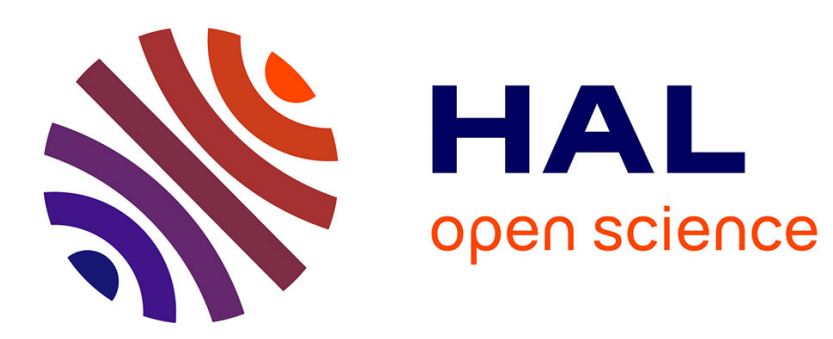

\title{
Cross-field electron diffusion due to the coupling of drift-driven microinstabilities
}

\author{
Kentaro Hara, Sedina Tsikata
}

\section{To cite this version:}

Kentaro Hara, Sedina Tsikata. Cross-field electron diffusion due to the coupling of drift-driven microinstabilities. Physical Review E , 2020, 102 (2), pp.023202. 10.1103/PhysRevE.102.023202 . hal02917882

\section{HAL Id: hal-02917882 \\ https://hal.science/hal-02917882}

Submitted on 7 Jan 2021

HAL is a multi-disciplinary open access archive for the deposit and dissemination of scientific research documents, whether they are published or not. The documents may come from teaching and research institutions in France or abroad, or from public or private research centers.
L'archive ouverte pluridisciplinaire HAL, est destinée au dépôt et à la diffusion de documents scientifiques de niveau recherche, publiés ou non, émanant des établissements d'enseignement et de recherche français ou étrangers, des laboratoires publics ou privés. 


\title{
Cross-field electron diffusion due to the coupling of drift-driven microinstabilities
}

\author{
Kentaro Hara $\mathbb{1}^{*}$ \\ Department of Aeronautics and Astronautics, 496 Lomita Mall, Stanford University, Stanford, California 94305, USA
}

Sedina Tsikata $\oplus^{\dagger}$

ICARE UPR 3021, Centre National de la Recherche Scientifique (CNRS), Orléans, France

(Received 29 December 2019; revised 23 May 2020; accepted 10 July 2020; published xxxxxxxxxx)

\begin{abstract}
In this paper, the nonlinear interaction between kinetic instabilities driven by multiple ion beams and magnetized electrons is investigated. Electron diffusion across magnetic field lines is enhanced by the coupling of plasma instabilities. A two-dimensional collisionless particle-in-cell simulation is performed accounting for singly and doubly charged ions in a cross-field configuration. Consistent with prior linear kinetic theory analysis and observations from coherent Thomson scattering experiments, the present simulations identify an ion-ion two-stream instability due to multiply charged ions (flowing in the direction parallel to the applied electric field) which coexists with the electron cyclotron drift instability (propagating perpendicular to the applied electric field and parallel to the $E \times B$ drift). Small-scale fluctuations due to the coupling of these naturally driven kinetic modes are found to be a mechanism that can enhance cross-field electron transport and contribute to the broadening of the ion velocity distribution functions.
\end{abstract}

DOI: 10.1103/PhysRevE.00.003200

\section{INTRODUCTION}

Electron diffusion across magnetic field lines plays an important role in a variety of contexts, including fusion, astrophysical, ionospheric, and cross-field plasma discharges. Partially magnetized plasmas, where ions are nonmagnetized and electrons are magnetized, exhibit enhanced electron mobility, i.e., reduced electron confinement, in the direction across the magnetic field lines [1,2]. Plasma turbulence is of critical importance for understanding transport of charged species [3]. A number of key kinetic instabilities have been investigated in the literature in the context of electron transport, including, but not limited to, the electron cyclotron drift instability (ECDI) [4-8], modified two-stream instability (MTSI) [9,10], and ion acoustic instability (IAI) [11-13].

Although electron diffusion across the magnetic field lines can be caused by a plasma wave in the $E \times B$ direction, microturbulence may be driven not only by one type of linear instability but by the nonlinear interaction of multiple linear instabilities [14]. In a laboratory cross-field discharge, theoretical and numerical studies identified the plasma waves driven by the ECDI [15-17] and these results were subsequently supported by coherent Thomson scattering experiments [18-21]. In recent years, an increasing number of numerical studies have been undertaken by several groups revisiting this instability [22-26] and its role in transport.

One of the key experimental results in Ref. [20] was the detection of a plasma wave in the cross-field direction (parallel to the applied electric field), exhibiting a spatial scale similar

\footnotetext{
*kenhara@stanford.edu

†sedina.tsikata@cnrs-orleans.fr
}

to that of the ECDI observed primarily in the $E \times B$ direction. Subsequent linear kinetic theory analyses revealed that such cross-field oscillations observed in experiments can be initiated by the ion-ion two-stream instability (IITSI) [27-31] due to the presence of singly and doubly charged ion streams. Generally, mode coupling of different instability mechanisms plays an important role in plasma transport, particularly in the nonlinear saturation phase of instabilities. However, it is difficult to evaluate the effects of such mode coupling on electron transport, e.g., current density, using diagnostic tools or linear theories. The linear growth rate denotes how fast an instability develops but does not account for how large its amplitude ultimately becomes, i.e., at what level the nonlinear saturation occurs. High-fidelity plasma simulations are therefore of critical importance to investigate nonlinear dynamics of coupled plasma instabilities and the corresponding electron transport.

This paper analyzes the microturbulence that develops due to the mode coupling between the IITSI and ECDI in a lowtemperature magnetized plasma. Theory and simulation of the kinetic instability that results from the interaction of multiple ion streams interacting with electron cyclotron dynamics are reviewed in Secs. II and III, respectively. Section IV discusses the plasma properties, and in particular, the observation of enhanced cross-field electron transport and modification of the ion distribution function resulting from the mode coupling.

\section{KINETIC INSTABILITIES}

Let us consider a partially magnetized plasma where an external electric field is applied in the $x$ direction and a magnetic field is applied in the $z$ direction, as shown in Fig. 1. 


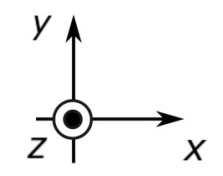

$n_{e}=n_{i}^{+}+2 n_{i}^{2+}$

$\alpha=\frac{2 n_{i}^{2+}}{n_{e}}$
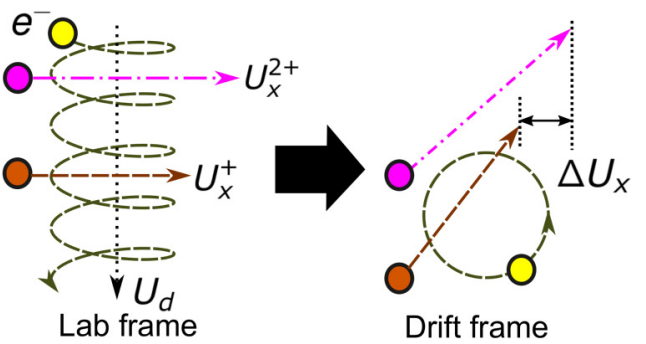

FIG. 1. A partially magnetized plasma where a DC electric field component and an external magnetic field are applied in the $x$ and $z$ directions, respectively. The difference between the singly charged ion velocity $U_{i}^{+}$and the doubly charged ion velocity $U_{i}^{2+}$ in the $x$ direction is denoted by $\Delta U_{x}$. Gyrating electrons move with an azimuthal drift, $U_{d}$, in the $-y$ direction.

Nonmagnetized ions are considered and are electrostatically accelerated in the $x$ direction while an electron drift, $U_{d}$, is formed in the $-y$ direction. Here, two cold ion streams are considered in the $x$ direction such that $n_{e}=n_{i}^{+}+2 n_{i}^{2+}$, where $n_{e}$ is the electron density, $n_{i}^{+}$is the singly charged ion density, and $n_{i}^{2+}$ is the doubly charged ion density. Here, $\alpha=2 n_{i}^{2+} / n_{e}$ is introduced, i.e., $n_{i}^{+} / n_{e}=1-\alpha$.

\section{A. Theory: Dispersion relation}

Assuming for the purposes of this study that the dynamics along the magnetic field are negligible $\left(k_{z}=k_{\|}=0\right)$, the twodimensional dispersion relation in the $x-y$ plane, accounting for two cold ion species and magnetized electrons [7], can be written as

$$
\begin{aligned}
& \left(k_{\perp} \lambda_{D}\right)^{2}\left[1-\frac{(1-\alpha) \omega_{p i}^{2}}{\left(\omega-\mathbf{k} \cdot \mathbf{U}_{i}^{+}\right)^{2}}-\frac{\alpha \omega_{p i}^{2}}{\left(\omega-\mathbf{k} \cdot \mathbf{U}_{i}^{2+}\right)^{2}}\right] \\
& +1-I_{0}(b) \exp (-b)+\sum_{n=1}^{\infty} \frac{2 \omega^{2} I_{n}(b) \exp (-b)}{\left(n \omega_{B}\right)^{2}-\omega^{2}}=0,
\end{aligned}
$$

where $k_{\perp}^{2}=k_{x}^{2}+k_{y}^{2}, \lambda_{D}=\left[\epsilon_{0} k_{B} T_{e} /\left(e^{2} n_{0}\right)\right]^{1 / 2}$ is the Debye length, $\omega_{p i}=\left[e^{2} n_{0} /\left(m_{i} \epsilon_{0}\right)\right]^{1 / 2}$ is the ion plasma frequency (here, $\epsilon_{0}$ is the vacuum permittivity, $k_{B}$ is the Boltzmann constant, $T_{e}$ is the electron temperature, $e$ is the elementary charge, $n_{0}$ is the plasma density, and $m_{i}$ is the ion mass), $\omega$ and $\mathbf{k}$ are the frequency and wave vector, $\mathbf{U}_{i}^{s}$ is the ion bulk velocity for species $s=+$ and $2+$ corresponding to singly and doubly charged ions, respectively, $b=\left(k_{\perp} r_{L}\right)^{2}$, $r_{L}=v_{\perp} / \omega_{B}$ is the Larmor radius [here, $v_{\perp}$ is assumed to be the electron thermal velocity $v_{\mathrm{th}, e}=\left(k_{B} T_{e} / m_{e}\right)^{1 / 2}, \omega_{B}=$ $e B / m_{e}$ is the electron gyrofrequency, $B$ is the magnetic field amplitude, and $m_{e}$ is the electron mass], and $I_{n}$ is the modified Bessel function of $n$th kind. $I_{n}(b) \exp (-b)$ is also known as the scaled modified Bessel function.

As shown in Fig. 1, the presence of an electron drift, e.g., $E \times B$ drift, can be accounted for by shifting the system to the frame of the electron drift. Here, $\mathbf{k} \cdot \mathbf{U}_{i}^{s}=k_{x} U_{x}^{s}-k_{y} U_{d}$, where $U_{x}$ is the drift parallel to the applied electric field and $U_{d}$ is the electron drift in the $y$ direction. The wave frequency can be shifted by $\omega-k_{x} U_{x}^{+}+k_{y} U_{y}$ and Eq. (1) can be written

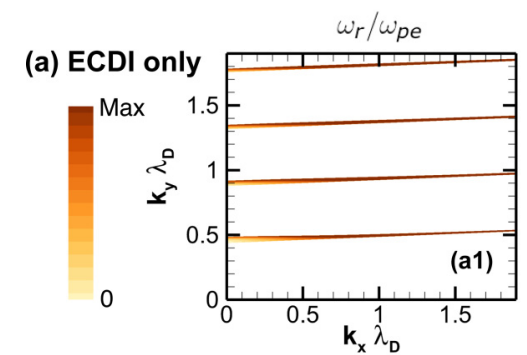

(b) IITSI only
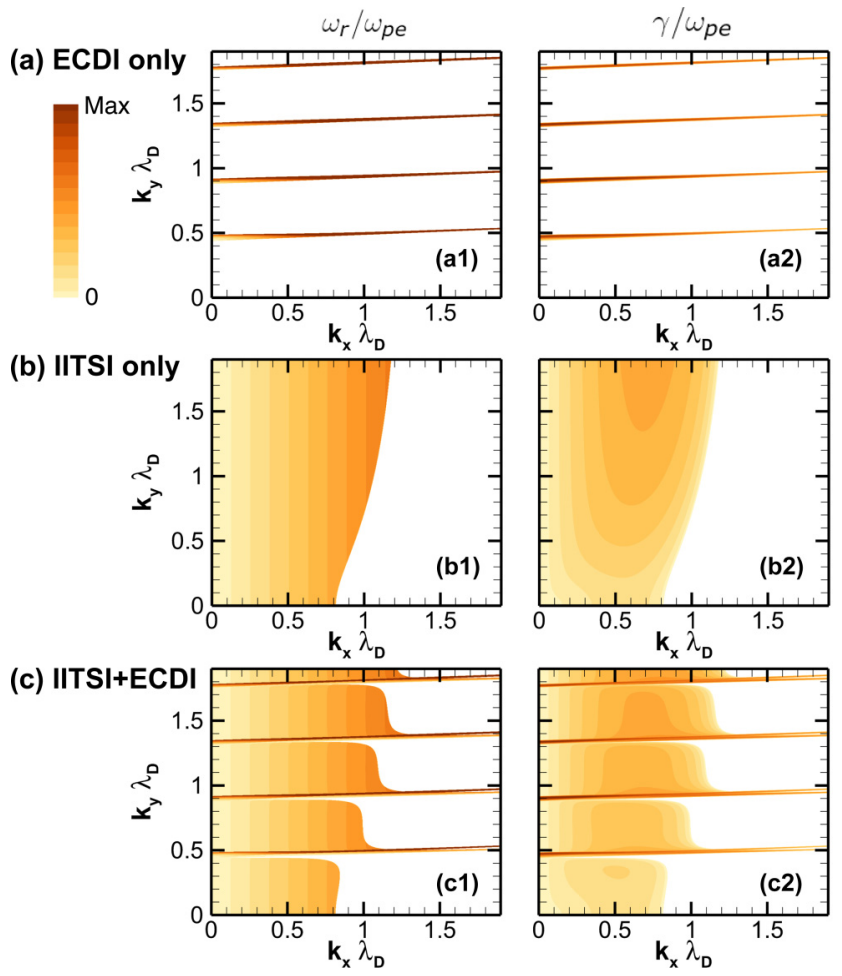

FIG. 2. Instabilities generated in a 2D partially magnetized plasma. (a) Electron cyclotron drift instability (ECDI) due to an electron drift in the $y$ direction, where $\tilde{U}_{d}=0.239$, assuming only singly charged ions, i.e., $\alpha=0$. (b) Ion-ion two-stream instability (IITSI) due to the mixture of singly and doubly charged ions, where $\Delta \tilde{U}_{x}=3.2 \times 10^{-3}$ and $\tilde{U}_{d}=0$. (c) Coexisting ECDI and IITSI. Maximum value of color map is 0.002 for $\omega_{r} / \omega_{p e}$ (left) and 0.001 for $\gamma / \omega_{p e}$ (right). Xenon ions are considered. Here, $B=150 \mathrm{G}$, $T_{e}=25 \mathrm{eV}, n_{0}=2 \times 10^{17} \mathrm{~m}^{-3}$, and $U_{x}^{+}=16 \mathrm{~km} / \mathrm{s}$.

in a normalized form as

$$
\begin{aligned}
& \tilde{k}_{\perp}^{2}\left[1-\frac{\mu(1-\alpha)}{\tilde{\omega}^{2}}-\frac{\mu \alpha}{\left(\tilde{\omega}-\tilde{k}_{x} \Delta \tilde{U}_{x}\right)^{2}}\right]+1-I_{0}(b) \exp (-b) \\
& \quad+\sum_{n=1}^{\infty} \frac{2\left(\tilde{\omega}+\tilde{k}_{x} \tilde{U}_{x}^{+}-\tilde{k}_{y} \tilde{U}_{d}\right)^{2} I_{n}(b) \exp (-b)}{\left(n \tilde{\omega}_{B}\right)^{2}-\left(\tilde{\omega}+\tilde{k}_{x} \tilde{U}_{x}^{+}-\tilde{k}_{y} \tilde{U}_{d}\right)^{2}}=0
\end{aligned}
$$

where $\mu=m_{e} / m_{i}$ is the electron-to-ion mass ratio and $\Delta U_{x}=$ $U_{x}^{2+}-U_{x}^{+}$is the difference between the doubly and singly charged ion velocities in the cross-field direction. The tilde quantities denote normalized parameters. Time, space, and velocity are normalized with respect to the electron plasma frequency, $\omega_{p e}=\left[e^{2} n_{0} /\left(m_{e} \epsilon_{0}\right)\right]^{1 / 2}$, Debye length, $\lambda_{D}$, and electron thermal speed, $v_{\text {th }, e}$, respectively.

The dispersion relation of the 2D ECDI when $\alpha=0$ (singly charged ions only) and $U_{d} \neq 0$ is shown in Fig. 2(a). The maximum growth rate is located near $k_{x}=0$. The ECDI dispersion relation at $k_{x}=0$ is shown in Fig. 3 (cf. Ref. [7]). The 2D dispersion relation yields a resonance condition for the ECDI, namely, $\tilde{k}_{y} \tilde{U}_{d}=n \tilde{\omega}_{B}$, where $n>0$. Note that the current-carrying ion-acoustic instability can be derived in the limit of zero magnetic field (i.e., $b \rightarrow \infty$ ) and singly charged ions (i.e., $\alpha=0$ ) [32]. 


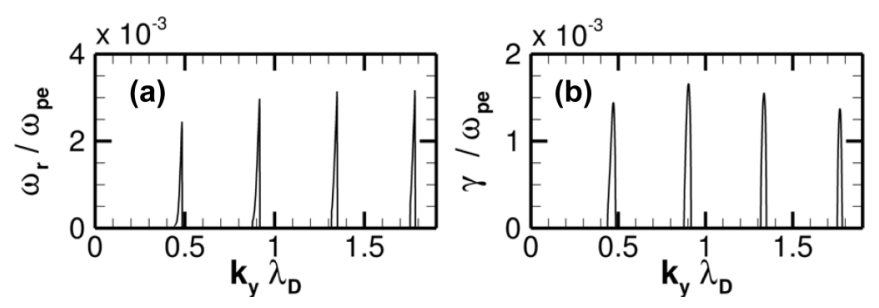

FIG. 3. ECDI dispersion relation at $k_{x} \lambda_{D}=0$ from Fig. 2: (a) real part of the frequency and (b) growth rate.

Figure 2(b) shows the case where the electron drift is absent, i.e., $U_{d}=0$, and $\alpha$ is set at 0.5 as an illustrative case. The unstable roots (resonant condition) of the IITSI can be found at $\tilde{k}_{x}<\sqrt{\mu} / \Delta \tilde{U}_{x}=O(1)$. The magnetized electron contribution becomes small under this condition, reducing Eq. (2) to a dispersion relation of a two-stream instability. Since $\tilde{U}_{x}^{+}$is a few orders of magnitude smaller than $\tilde{U}_{d}$, the ECDI-type resonance with the axial velocity is unlikely to be observed.

Figure 2(c) illustrates the coexistence of the ECDI and IITSI. The resonances of the ECDI in 2D (narrow lobes at discrete $k_{y}$ values and present for all $k_{x}$ ) are apparent, along with the IITSI solutions as shown in Fig. 2(b). It can be seen that the ECDI growth rates are larger than the IITSI growth rates in the present $2 \mathrm{D}$ configuration since the ECDI exhibits discrete resonance-type solutions. In addition, the presence of the ECDI lobes in Fig. 2(c), while not affecting the observed IITSI mode frequencies, does reshape the unstable regions corresponding to the IITSI.

\section{B. Observations from experiments}

The IITSI under study in this work is distinct from the ECDI, not only with regard to the instability mechanism, but also in terms of the spatial localization in laboratory cross-field discharges such as Hall effect thrusters and planar magnetrons.

In coherent Thomson scattering experiments [18,20], wave identification is performed through the measurement of electron density fluctuations. The diagnostic technique allows for the measurement of such fluctuations not only at different length scales but also along different directions, e.g., aligned primarily with the $E \times B$ drift in studies of the ECDI or primarily along the applied electric field in studies of the IITSI

These experiments have provided evidence (i) that both ECDI and IITSI modes, although different in their nature of excitation, are associated with density fluctuations of similar spatial scales, i.e., electron Larmor radius scales (on the order of $1 \mathrm{~mm}$ ), (ii) that the fluctuations driven by ECDI (i.e., $k_{y} \neq$ 0 ) are strongest in the region of maximum $E \times B$ velocity and detectable further downstream due to convection, and (iii) that the fluctuations driven by IITSI (i.e., $k_{x} \neq 0$ ) are detectable not only in a spatial region overlapping the largest-amplitude ECDI fluctuations but also over a very large axial region over which the ions are accelerated. While the plasma density fluctuations are evident from measurements, it is difficult to quantify the effects of such instability-driven plasma waves on electron transport. As the following discussions will show, the present numerical study captures such features and clarifies the dynamics of each mode.

\section{PARTICLE-IN-CELL SIMULATION OF THE PARTIALLY MAGNETIZED PLASMA}

In the present paper, we focus on the physics of the coupling between ECDI and IITSI modes within the same computational framework in the literature. The computational setup to study the $E \times B$ discharge is identical to that originally proposed by Boeuf and Garrigues [24] and used as a benchmarking test case [33]. The 2D particle-in-cell (PIC) simulation used in this paper (explicit PIC with particle and domain decomposition) is described in Ref. [33] and has been compared with other PIC codes.

Ionization occurs upstream where the electrons are trapped by the magnetic fields. The crossed electric and magnetic fields generate an $E \times B$ drift for electrons (the source of the ECDI) and ions are accelerated electrostatically (the source of IISTI in the presence of singly and doubly charged ion streams). The ionization rate is constant in time, leading to a constant ion current density. In steady state, $\boldsymbol{\nabla} \cdot\left(\mathbf{j}_{i 1}+\mathbf{j}_{i 2}\right)=$ $e\left(S_{i 1}+2 S_{i 2}\right)$, where $\mathbf{j}_{i k}$ is the ion current density and $S_{i k}$ is the ionization rate for singly $(k=1)$ and doubly $(k=$ 2 ) charged ions. Defining $\alpha_{0}$ to be the fraction of doubly charged ion current density, the individual source terms are assigned as $S_{i 1} / S_{i}=1-\alpha_{0}$ and $S_{i 2} / S_{i}=\alpha_{0} / 2$, where $S_{i}(x)=$ $S_{0} \cos \left[\pi\left(x-x_{M}\right) /\left(x_{2}-x_{1}\right)\right]$ is the total ionization rate, $x_{1}=$ $0.25 \mathrm{~cm}, x_{2}=1 \mathrm{~cm}, x_{M}=\left(x_{1}+x_{2}\right) / 2$, and $S_{0}$ is adjusted so that the total ion current density is $400 \mathrm{~A} / \mathrm{m}^{2}$. Xenon ions are considered. Note that $\alpha_{0}$ is similar but not identical to $\alpha$ in Sec. II.

The domain size is $L_{x}=2.5 \mathrm{~cm}$ and $L_{y}=1.28 \mathrm{~cm}$ in the $x$ and $y$ direction, respectively. The magnetic field is set to $B(x, y)=B_{\max }+B_{0} \xi(x)$, where $\xi(x)=1-\exp [-0.5\{(x-$ $\left.\left.\left.x_{L}\right) / \sigma_{b}\right\}^{2}\right]$ and $B_{0}=\left(B_{a}-B_{\max }\right) / \xi(0)$ if $x<x_{L}$ and $B_{0}=$ $\left(B_{c}-B_{\max }\right) / \xi\left(x_{L}\right)$ if $x \geqslant x_{L}$. Here, $x_{L}=0.75 \mathrm{~cm}, \sigma_{b}=0.625$ $\mathrm{cm}, B_{\max }=100 \mathrm{G}, B_{a}=60 \mathrm{G}$, and $B_{c}=10 \mathrm{G}$. Intermolecular collisions, neutral atom dynamics, and transport in the $z$ direction are neglected. The potential drop between $x=0 \mathrm{~cm}$ and $2.4 \mathrm{~cm}$ is kept constant at $200 \mathrm{~V}[24,33]$. The electrons are reinjected randomly in the $y$ direction at $x=2.4 \mathrm{~cm}$ to satisfy charge neutrality in the system, i.e., $\Gamma_{e c}=\Gamma_{e a}-\Gamma_{i 1 a}-2 \Gamma_{i 2 a}$, where $\Gamma_{e c}$ is the number of electrons reinjected from the cathode plane, and $\Gamma_{e a}, \Gamma_{i 1 a}$, and $\Gamma_{i 2 a}$ are the number of electrons, singly charged ions, and doubly charged ions absorbed at the anode plane, respectively.

The average number of particles per cell is 250 in the steady state, which shows satisfactory convergence based on the study in Ref. [33]. The grid size is $50 \mu \mathrm{m}$ in both directions; i.e., the number of cells is 500 and 256 in the $x$ and $y$ directions, respectively. The simulation utilizes a message passing interface (MPI) and the Poisson equation is solved using Hypre, a linear algebra library.

\section{RESULTS}

The effects of doubly charged ions are investigated by varying $\alpha_{0}$ from $0 \%$ to $25 \%$, which is the range of $\mathrm{Xe}^{2+}$ observed in $E \times B$ discharges [34,35]. 

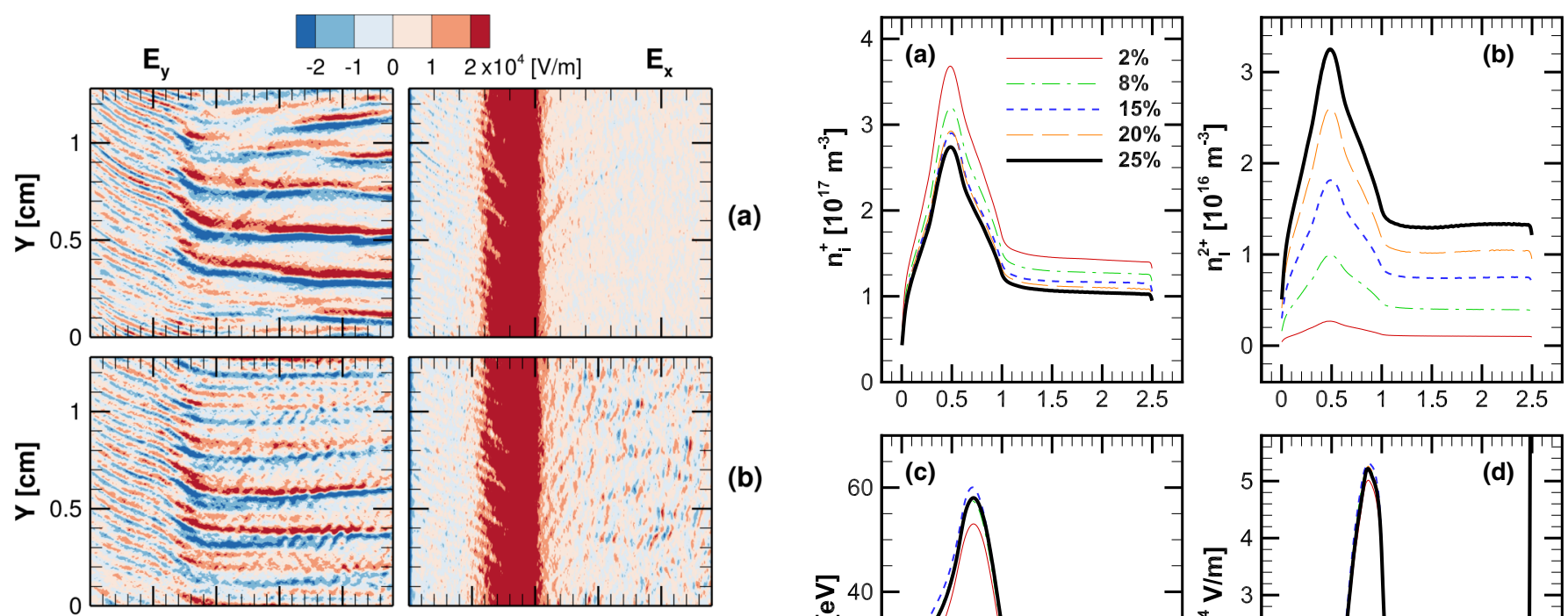

(b)
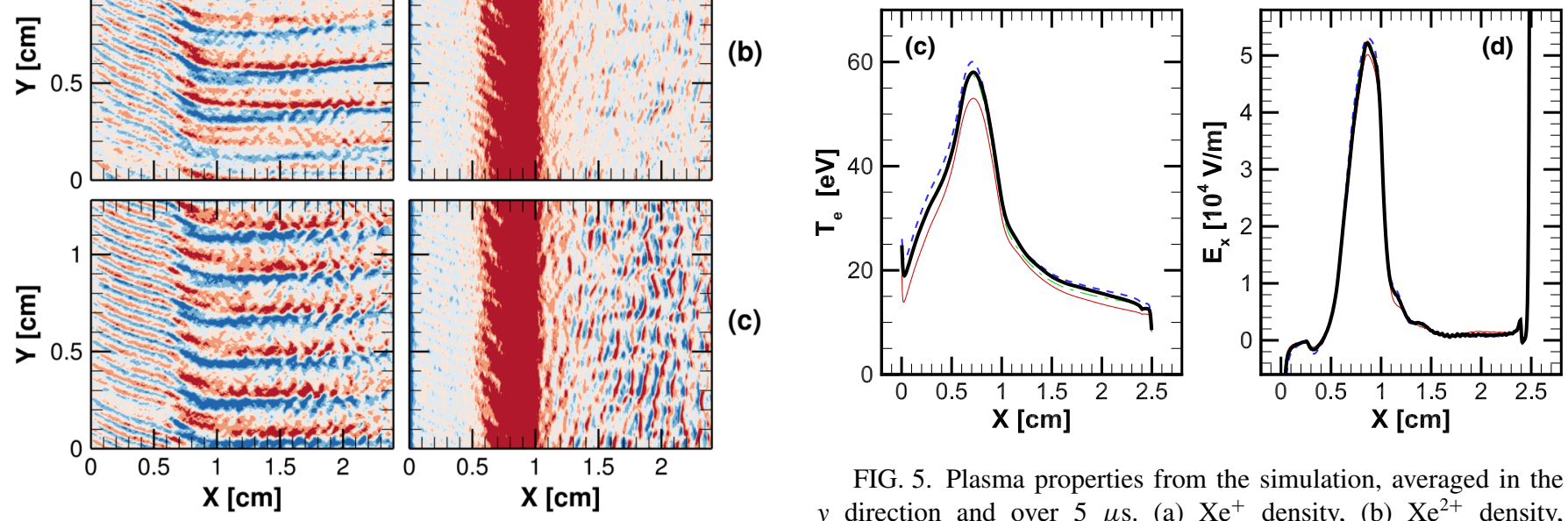

FIG. 5. Plasma properties from the simulation, averaged in the $y$ direction and over $5 \mu \mathrm{s}$. (a) $\mathrm{Xe}^{+}$density, (b) $\mathrm{Xe}^{2+}$ density, (c) electron temperature, and (d) axial electric field. Ionization rate and magnetic field profiles were fixed while varying the ratio of doubly charged ionization to the total ionization rate, $\alpha_{0}$.

instabilities in the presence of singly and doubly charged ion streams. (a) $\alpha_{0}=0$, i.e., singly charged ions only, illustrating the ECDI in the azimuthal direction, (b) $\alpha_{0}=10 \%$ (moderate-amplitude IITSI), and (c) $\alpha_{0}=20 \%$ (large-amplitude IITSI). The axially modulated electric field develops as the fraction of doubly charged ions increases. The color bar is saturated, particularly for $E_{x}$, to allow for visualization of the plasma waves in the downstream region.

In this work, a fixed ionization rate is assumed to allow the plasma instabilities to evolve naturally and reach steady state without the need to run the simulation much longer, i.e., to resolve the slow neutral dynamics. The plasma waves driven by the instabilities achieve steady state after $10 \mu$ s and the simulations are run up to $30 \mu \mathrm{s}$ (or longer) to ensure that the plasma state does not diverge. The same strategy was validated in Ref. [33] in simulations of authors from several groups. While oscillations on the order of $200 \mathrm{kHz}$ (also present in benchmarking simulations in Ref. [33]) are observed in $\alpha_{0} \leqslant 15 \%$, such oscillations are not seen in the $\alpha_{0}=20 \%$ and $25 \%$ cases. Investigation of the low-frequency oscillations requires simulations that self-consistently model ionization and collisions, which is reserved for future work.

\section{A. Coexistence of IITSI and ECDI}

Figure 4 shows the instantaneous electric fields, $E_{y}$ and $E_{x}$, at $t=18 \mu \mathrm{s}$, in the steady state. The results with only $\mathrm{Xe}^{+}$, shown in Fig. 4(a), are consistent with Ref. [33]. The azimuthal plasma fluctuations, i.e., $E_{y}$, driven by the ECDI are advected downstream. It can be seen from Figs. 4(b) and 4(c) that when $\mathrm{Xe}^{2+}$ is added, a cross-field (axial) mode in the $x$ direction emerges at $x>1 \mathrm{~cm}$, where the ions are accelerated downstream. The amplitude of $E_{x}$ in the downstream region increases as the doubly charged ion contribution, $\alpha_{0}$, increases. The axial fluctuation of $E_{y}$ is also evident. The dominant wavelength of the $x$ fluctuation is approximately $1 \mathrm{~mm}$, which corresponds to $k_{x}=6200 \mathrm{rad} / \mathrm{m}$. Using the time-averaged, $y$-averaged plasma properties, $\tilde{k}_{x}=k_{x} \lambda_{D} \approx$ $0.5-0.6$, which is in good agreement with the theoretical dispersion relation in Fig. 2.

The results of Fig. 4 shed light on aspects concerning the two instabilities not previously accessible via experiments and linear kinetic theory analysis [20,32]. Figure 4 shows that for axial positions which coincide with those of the experimental measurements (about $x-x_{L} \geqslant 1 \mathrm{~cm}$ ), both the ECDI field modulation (along $y$ ) and the IITSI field modulation (along $x$ ) are present. Additionally, the present simulations elucidate the regions in which different instabilities are created. The ECDI is driven in the region of the fastest electron drift (approximately where $E_{x} / B_{z}$ is largest) as expected, while the IITSI fully develops once the velocity difference $\Delta U_{x}$ between the singly and doubly charged ion streams becomes large enough after acceleration. As previous experiments were only performed outside the channel in the downstream region due to restricted laser beam access, the present simulation results provide information on how the instabilities evolve in a multidimensional configuration.

The plasma properties averaged over $5 \mu$ s and the $y$ direction are shown in Fig. 5. The decrease of $\mathrm{Xe}^{+}$density and 

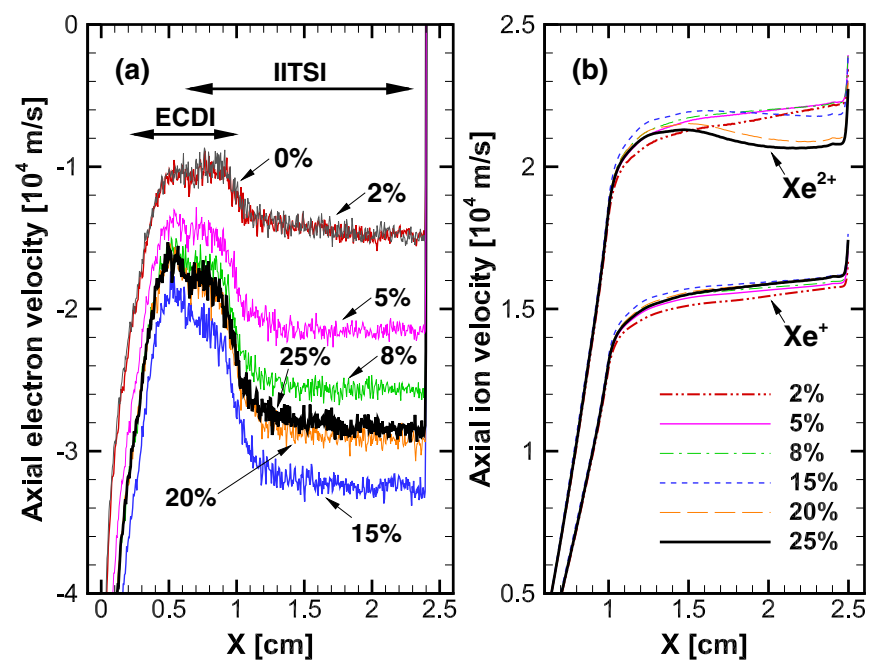

FIG. 6. Enhanced cross-field transport of electrons due to the kinetic instabilities driven by doubly charged ions. The plasma properties are averaged in the $y$ direction and over $5 \mu \mathrm{s}, 200$ sampling. (a) Electron velocity and (b) ion $\left(\mathrm{Xe}^{+}\right.$and $\left.\mathrm{Xe}^{2+}\right)$ velocities for various values of $\alpha_{0}$. Note that $x=2.4 \mathrm{~cm}$ is where the electrons are injected in the simulation.

increase of $\mathrm{Xe}^{2+}$ density can be observed from Figs. 5(a) and 5(b), as expected. It should be noted that there is a slight increase in the $\mathrm{Xe}^{2+}$ density in the downstream region, which is due to the deceleration caused by ion trapping due to the wave-particle interaction. This will be discussed in more detail shortly. Figures 5(c) and 5(d) illustrate that doubly charged ions do not significantly alter the electron temperature and axial electric field.

\section{B. Cross-field electron and ion transport}

Figure 6(a) shows the enhancement of cross-field electron transport by the presence of doubly charged ions in addition to singly charged ions. Compared to cases where $\alpha_{0}$ is small, e.g., $\alpha_{0} \leqslant 2 \%$, the cross-field electron transport is enhanced by up to approximately $90 \%$ at larger $\alpha_{0}$ cases. Considering a drift-diffusion approximation for the electron transport in the cross-field direction,

$$
u_{e, \perp}=-\mu_{\perp}\left(E_{\perp}+\frac{1}{e n_{e}} \nabla_{\perp} p_{e}\right)
$$

where $\mu_{\perp}$ is the cross-field mobility and $p_{e}$ is the electron pressure. Since the time-averaged plasma properties, such as $E_{\perp}\left(=E_{x}\right), n_{e}$, and $T_{e}$ (see Fig. 5), are not modified significantly by $\mathrm{Xe}^{2+}$, a large $\left|u_{e, \perp}\right|$ indicates that the effective cross-field mobility has indeed increased. While anomalous electron transport models have been proposed, such as the Bohm diffusion $\mu_{\perp}=(16 B)^{-1}$, the present PIC results suggest that $\mu_{\perp}$ is dependent on the doubly charged ion fraction, $\alpha_{0}$. Figure 4(c) shows that the amplitude of the plasma wave in the downstream region becomes approximately the same in the $x$ and $y$ directions, despite the fact that the growth rate of the ECDI is an order of magnitude larger than that of the IITSI as illustrated in Sec. II. The electric field fluctuations in both directions enhance the cross-field transport, but not merely by randomizing the electron motions, which can be inferred from the fact that the electron temperature is not drastically changed as shown in Fig. 5(c).

The enhanced electron transport across the magnetic field lines is correlated with the coexistence of the ECDI and IITSI. These two instabilities are generated and interact as follows: (i) The ECDI is created in the upstream region, i.e., $x \sim 0.5$ $\mathrm{cm}$. The plasma wave is generated due to the resonance at $k_{y} \lambda_{D} \approx 0.9$, where the growth rate is at maximum. However, there is a transition to a larger wavelength mode at $x>0.6 \mathrm{~cm}$. In this region, it is observed that $k_{y} \lambda_{D} \approx 0.3$, which is possibly due to the physical phenomena not taken into account in the theory. (ii) At $0.7 \mathrm{~cm}<x<1 \mathrm{~cm}$, the ECDI and IITSI can coexist since an azimuthal electron drift exists and the velocity difference between $\mathrm{Xe}^{+}$and $\mathrm{Xe}^{2+}, \Delta U_{x}$, increases, which can be seen from Fig. 6(b). (iii) In the downstream region, i.e., $x \geqslant 1 \mathrm{~cm}$, since the azimuthal drift is small, the ECDI is unlikely to occur. Instead, the increasing nonzero $\Delta U_{x}$ further excites the IITSI. Since the plasma wave generated by the ECDI upstream is advected downstream, the IITSI is first initiated in the presence of the $k_{y}$ component driven by the ECDI.

It can be observed from Fig. 6(a) that the electron bulk velocity is relatively constant between $x \in[0.5 \mathrm{~cm}, 1 \mathrm{~cm}]$ for the small $\alpha_{0}$ cases ( 0 and $2 \%$ ), while its magnitude increases over the same spatial interval (seen in the sloping trend developing over this region) in the presence of doubly charged ions $\left(\alpha_{0}\right.$ exceeding $\left.2 \%\right)$. This indicates that the electron mobility is modified due to the presence of doubly charged ions. As can be seen from Fig. 6(b), in this region, the difference in the axial ion bulk velocities is nonzero, e.g., $\Delta U_{x}=1-4$ $\mathrm{km} / \mathrm{s}$, and the azimuthal electron drift is nonzero, e.g., $U_{d} \approx$ $10^{6} \mathrm{~m} / \mathrm{s}$. With these features taken into account, it is expected that both ECDI and IITSI modes will develop simultaneously within this region, as discussed in Fig. 2(c).

The consequence of the cross-field IITSI due to the multiple ion streams (here, singly and doubly charged ions) is that the streaming ions with different velocities thermalize and equilibrate. This is apparent in Fig. 6(b) where the cross-field bulk velocity of the $\mathrm{Xe}^{2+}$ decreases for the $\alpha_{0}=20 \%$ and $25 \%$ cases in the downstream region $(x \geqslant 1.5 \mathrm{~cm})$. The crossfield plasma wave propagates with its own phase velocity and traps, i.e., decelerates and heats, the doubly charged ions, which is similar to the instabilities that occur within the plasma sheath [31]. The nonlinear trapping of $\mathrm{Xe}^{2+}$ coincides with the inverse tendency in the electron transport from $\alpha_{0}=$ $15 \%$ to $\alpha_{0}=20 \%$ and 25\%, as can be seen from Fig. 6(a). The ion velocity distribution functions (VDFs) will be shown later.

The IITSI growth rate increases monotonically as $\alpha_{0}$ increases for $\alpha_{0} \in[0,0.25]$. From an order of magnitude analysis, $\gamma / \omega_{p e} \leqslant O\left(10^{-4}\right)$ and the characteristic time for the IITSI to grow, $\tau \propto \gamma^{-1}$, is larger than $0.1 \mu \mathrm{s}$. It is to be noted that the IITSI in the present simulation is a convective instability. Since the ions are advected in the $x$ direction with a speed, $v$, on the order of $10 \mathrm{~km} / \mathrm{s}$, the characteristic distance for the IITSI to grow is $L=v \tau$. When the growth rate of the IITSI is small, i.e., for a small $\alpha_{0}, L$ is large. As $\alpha_{0}$ increases, the IITSI growth rate becomes large; thus, $L \propto \gamma^{-1}$ correspondingly decreases. Simultaneously, the plasma wave amplitude in the 


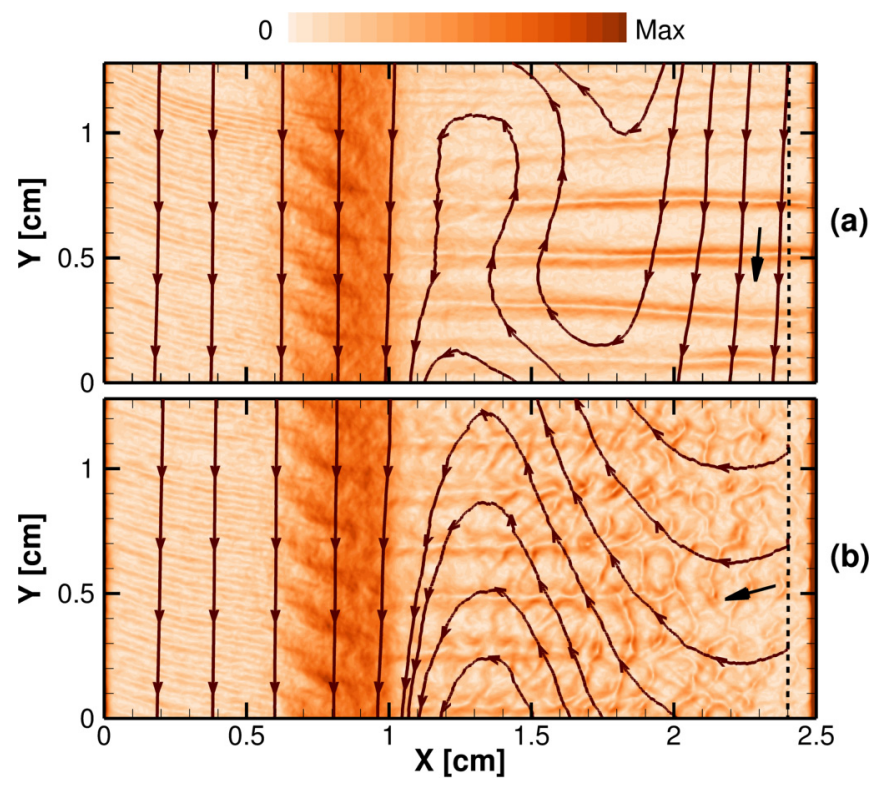

FIG. 7. Electron streamlines averaged over $1 \mu$ s and instantaneous profile of the magnitude of electric fields, $|\mathbf{E}|=\sqrt{E_{x}^{2}+E_{y}^{2}}$ in Fig. 4, for the (a) ECDI and (b) ECDI and IITSI cases. Maximum value of $|\mathbf{E}|$ is $80 \mathrm{kV} / \mathrm{m}$. The vertical dashed line indicates the plane of electron injection. Arrows are shown to help the visualization of the electron streamline near the electron injection plane at $x=2.4$ $\mathrm{cm}$.

axial direction, or equivalently $E_{x}$, increases for a larger $\alpha_{0}$, as shown in Fig. 4. The characteristic length over which the IITSI grows becomes on the order of a few millimeters. This can be seen also from Fig. 6(b), where the deceleration of $\mathrm{Xe}^{2+}$, potentially due to the saturation of the axial wave, is apparent from $x>1.5 \mathrm{~cm}$ for $\alpha_{0}=20 \%$ and $25 \%$.

\section{Electron turbulent transport}

Figure 7 shows the effects of the multidimensional plasma wave structures on the electron streamline to investigate the enhanced cross-field electron transport. The streamline denotes the direction of the time-averaged electron current. The ECDI-only case in Fig. 7(a) corresponds to $\alpha_{0}=2 \%$ while Fig. 7(b), showing both ECDI and IITSI, corresponds to $\alpha_{0}=$ $20 \%$.

One of the most notable observations from Fig. 7 is the differences in electron streamline, i.e., direction of the electron flow, near the plane of electron injection at $x=2.4 \mathrm{~cm}$ (see the arrows in Fig. 7), despite the similarity of the averaged $E_{x}$ profiles, as shown in Fig. 5(d). The temporally and spatially averaged electron flux can be written as $\left\langle\Gamma_{e x}\right\rangle=\left\langle n_{e} E_{y}\right\rangle / B_{z}$ and $\left\langle\Gamma_{e y}\right\rangle=-\left\langle n_{e} E_{x}\right\rangle / B_{z}[36,37]$. Consider that plasma properties can be written as $Q=Q_{0}+Q^{\prime}$, where $Q_{0}$ and $Q^{\prime}$ denote the steady-state value and fluctuation of $Q=n_{e}, E_{x}, E_{y}$. Here, the electron flux in the cross-field $(x)$ direction can be given as

$$
\left\langle\Gamma_{e x}\right\rangle=\frac{\left\langle n_{e}^{\prime} E_{y}^{\prime}\right\rangle}{B_{z}}
$$

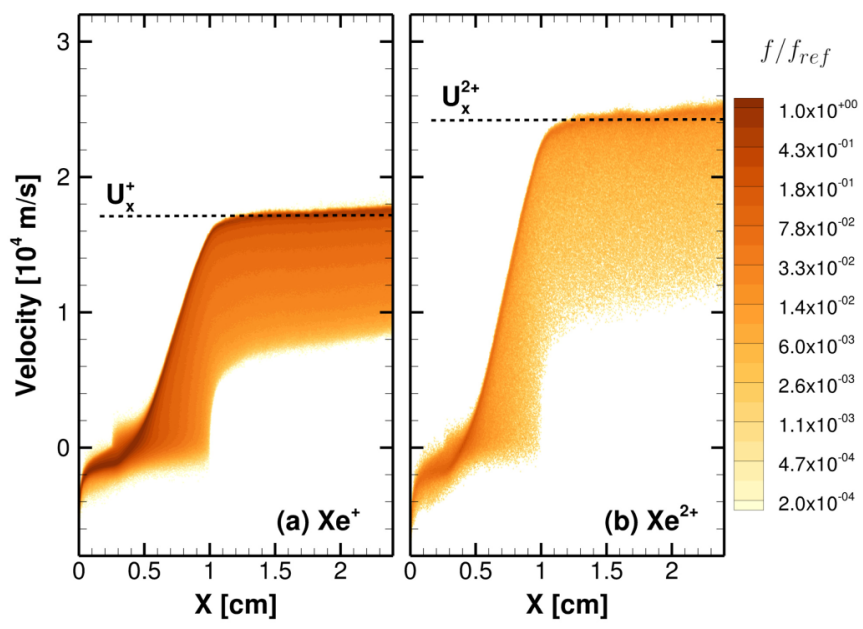

FIG. 8. The instantaneous ion velocity distribution function for $\alpha_{0}=2 \%$, averaged over the $y$ direction, for (a) $\mathrm{Xe}^{+}$and (b) $\mathrm{Xe}^{2+}$. The horizontal dashed lines indicate the corresponding ion velocity $U_{x}^{Z+}=\left(Z e V_{d} / m_{i}\right)^{1 / 2}$, where $Z$ is the number of charges. The reference VDF value for $\mathrm{Xe}^{+}$is chosen to be approximately the maximum value of $\mathrm{Xe}^{+}, f_{\text {ref }}=f_{\max }^{+}$. Additionally, $f_{\text {ref }}=0.1 f_{\max }^{+}$is used for the VDFs of $\mathrm{Xe}^{2+}$.

since $E_{y 0}=0$ taking the average of $E_{y}$ in the $y$ direction (cf. periodic boundary condition). The electron flux in the $y$ direction can be written as

$$
\left\langle\Gamma_{e y}\right\rangle=-\frac{n_{e 0} E_{x 0}}{B_{z}}-\frac{\left\langle n_{e}^{\prime} E_{x}^{\prime}\right\rangle}{B_{z}} .
$$

The angle bracket quantities in Eqs. (4) and (5) denote the turbulent contribution, i.e., fluctuation-based transport.

Figure 7(a) shows that $\left|\left\langle\Gamma_{e x}\right\rangle\right|<\left|\left\langle\Gamma_{e y}\right\rangle\right|$ within $x \in[2 \mathrm{~cm}$, $2.4 \mathrm{~cm}]$ where the electrons are injected. The injected electrons primarily flow in the $-y$ direction for the ECDI-only case, which is consistent with the $-E_{x 0} \times B_{z}$ drift. The finite $\left|\left\langle\Gamma_{e x}\right\rangle\right|$ indicates that azimuthal $E_{y}$ fluctuations $\left(k_{y} \neq 0\right)$ induce the electron transport across the magnetic field in the absence of collisions, as discussed in Eq. (4).

In contrast, in the presence of the coupled ECDI and IITSI as shown in Fig. 7(b), electrons adopt more axial trajectories in the $-x$ direction, indicating $\left|\left\langle\Gamma_{e x}\right\rangle\right|>\left|\left\langle\Gamma_{e y}\right\rangle\right|$ within $x \in[2$ $\mathrm{cm}, 2.4 \mathrm{~cm}]$. The amplitude of $E_{x}$ fluctuation increases and the $E_{y}$ fluctuations become multidimensional, i.e., $k_{x} \neq 0$ and $k_{y} \neq 0$, in the coupled ECDI and IITSI case, as shown in Fig. 4(c). This is further evidence that the cross-field electron transport is enhanced by small-scale plasma fluctuations due to the presence of the axial plasma wave $\left(k_{x} \neq 0\right)$ in addition to the azimuthal fluctuations $\left(k_{y} \neq 0\right)$. Note that the electrons are advected in the $+y$ direction at $x \in[1 \mathrm{~cm}, 1.7 \mathrm{~cm}]$ in Fig. 7. While such trajectories can be influenced by various drifts, including $E \times B$, diamagnetic, and gradient drifts [38], the cross-field electron flux is enhanced in the presence of singly and doubly charged ion streams, as shown in Fig. 6(a).

\section{Broadening of ion velocity distribution functions}

Figure 8 shows instantaneous ion velocity distribution functions (VDFs) averaged over the $y$ direction for both $\mathrm{Xe}^{+}$and $\mathrm{Xe}^{2+}$. Here, $\alpha_{0}=2 \%$. The particles are sampled 


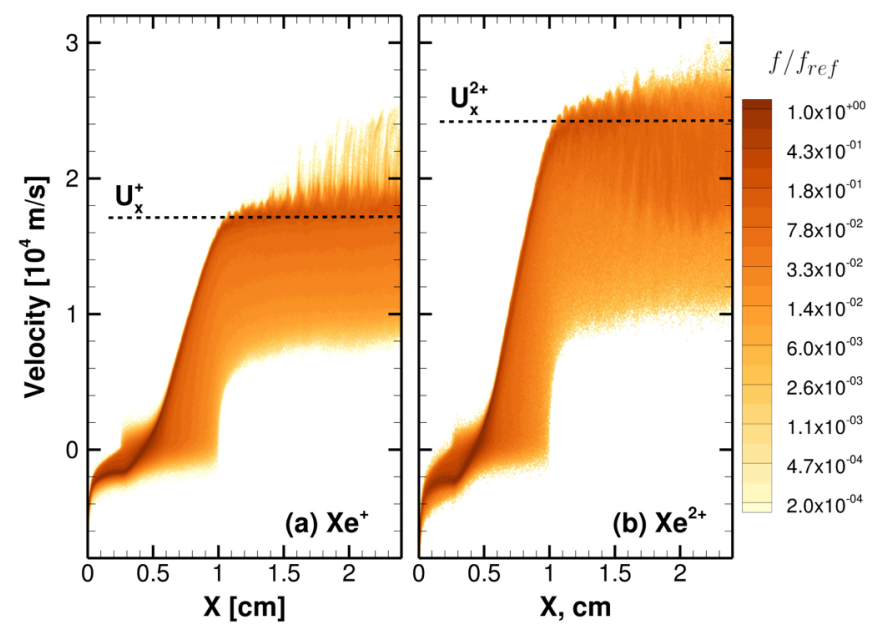

FIG. 9. Cross-field ion trapping observed in $\alpha_{0}=20 \%$ from the instantaneous ion velocity distribution function averaged over the $y$ direction. Color map is identical to Fig. 8.

into the discretized phase space, here $\Delta x=5 \times 10^{-5} \mathrm{~m}$ and $\Delta v=100 \mathrm{~m} / \mathrm{s}$. The ion bulk velocities obtained from the PIC simulation agree well with the values, $U_{i}^{+}$and $U_{i}^{2+}$, which assume a steady-state acceleration of ions across the discharge voltage, $V_{d}$. Here, $U_{x}^{+} \approx 1.7 \times 10^{4} \mathrm{~m} / \mathrm{s}$ and $U_{x}^{2+} \approx$ $2.4 \times 10^{4} \mathrm{~m} / \mathrm{s}$ assuming a potential drop of $V_{d}=200 \mathrm{~V}$.

As shown in Fig. 8, the ion VDFs have some spread in the velocity space due to the spatial profile of the ionization rate. Such a velocity spread, i.e., nonzero ion temperature, can damp the two-stream instabilities. The electron transport at $\alpha_{0} \leqslant 2 \%$ in our PIC simulation is indeed similar to that of the singly charged ion only case, i.e., $\alpha_{0}=0$, which is illustrated in Fig. 6(a).

Figure 9 shows the ion VDFs for $\alpha_{0}=20 \%$. While the ions form a beamlike structure for cases with smaller $\alpha_{0}$ (Fig. 8) since the $E_{x}$ fluctuation is small, by increasing the doubly charged ion contribution, ion trapping features now appear in both $\mathrm{Xe}^{+}$and $\mathrm{Xe}^{2+}$. The phase velocity of the plasma wave in the $x$ direction is between $U_{x}^{+}$and $U_{x}^{2+}$. Perturbation of $\mathrm{Xe}^{2+}$ by the axial plasma wave is observed in a wide range of $\alpha_{0}$ since some $\mathrm{Xe}^{2+}$ particles are already populated in the range of the wave velocity, $v_{\phi}$, which is between $U_{x}^{+}$and $U_{x}^{2+}$. The phase velocity can be estimated as $v_{\phi}=\omega / k_{x} \approx U_{x}^{+}+c_{s}$. However, without the axial plasma wave, there are virtually no $\mathrm{Xe}^{+}$ions in the range of $v_{\phi}>U_{x}^{+}$. Hence, the amplitude of the plasma wave must be large enough to perturb and start trapping $\mathrm{Xe}^{+}$ions. As can be seen from Fig. 9, the trapping of both $\mathrm{Xe}^{+}$and $\mathrm{Xe}^{2+}$ becomes visible at $\alpha_{0} \geqslant 20 \%$, which is consistent with the deceleration of doubly charged ion bulk velocity shown in Fig. 6(b). It can be considered that at this $\alpha_{0}$ value, $E_{x}$ (hence, the potential amplitude, $\phi_{0}$ ) becomes large enough such that

$$
\left|v_{\phi}-U_{x}^{Z+}\right| \geqslant\left(\frac{Z e \phi_{0}}{m_{i}}\right)^{1 / 2}
$$

where $v_{\phi}$ is the phase velocity of the wave and $U_{x}^{Z+}=$ $\left(Z e V_{d} / m_{i}\right)^{1 / 2}$ is the ion beam velocity for multiply charged ion states $Z=1$ and 2. The right-hand side of Eq. (6) is the trapping velocity of charged species. The results strongly indicate that the decrease in electron current from $\alpha_{0}=15 \%$ to $20 \%$, as shown in Fig. 6(a), is correlated with the ion trapping.

These findings provide better insight into the significance of some experimental results. Broadening of the $\mathrm{Xe}^{+}$ion distribution has been observed in laser-induced fluorescence measurements [39]. In the absence of any axial oscillations, the maximum ion velocity is $U_{i}^{+}$, limited by the applied DC voltage, as shown in Fig. 8. While some studies have attributed such high-energy ion formation to wave-riding effects $[22,40,41]$, where the discharge oscillation can generate ions whose energy is larger than the applied DC voltage, the IITSI due to the mixture of $\mathrm{Xe}^{+}$and $\mathrm{Xe}^{2+}$ can broaden the ion VDFs even in the absence of low-frequency discharge oscillations.

\section{DISCUSSION}

As the results discussed in this paper attest, the presence of the axially propagating IITSI, coupled to the azimuthal ECDI, can influence the level of electron transport. The doubly charged ion species concentration need only be low (2\% and above) for such effects to develop. The low threshold for the appearance of the IITSI, and its demonstrated effects on transport, suggest the importance of accounting for doubly charged ions in conventional low-temperature magnetized plasmas. Although we have opted to consider interaction between the two dominant ion streams in this study, triply charged xenon ions have been measured in some $E \times B$ discharges [42] and the presence of such species may be worth accounting for as well. The formation of axial plasma waves can also be critical for ion beam spreading in the transverse (radial) direction via ponderomotive forces [43,44] and would be expected to influence macroscopic behavior in low-temperature magnetized plasmas. Understanding how the small-scale turbulence affects the large-scale self-organization, e.g., rotating spokes [45], is reserved for future work.

While the simulations performed in this paper are in $2 \mathrm{D}$, here the 3D dispersion relation is discussed. The electron component in Eqs. (1) and (2) utilizes the 2D approximation $\left(k_{\|}=0\right)$, but can be updated to account for the 3D effects $\left(k_{\|} \neq 0\right)$. The 3D dispersion relation $[19,46]$ using normalized quantities can be written as

$$
\begin{aligned}
\tilde{k}^{2}\left[1-\frac{\mu(1-\alpha)}{\tilde{\omega}^{2}}-\frac{\mu \alpha}{\left(\tilde{\omega}-\tilde{k}_{x} \Delta \tilde{U}_{x}\right)^{2}}\right]+\bar{\xi}\left\{Z(\bar{\xi}) I_{0}(b) e^{-b}\right. \\
\left.\quad+\sum_{n=1}^{\infty}\left[Z\left(\xi^{+}\right)+Z\left(\xi^{-}\right)\right] I_{n}(b) \exp (-b)\right\}+1=0
\end{aligned}
$$

where $k^{2}=k_{\perp}^{2}+k_{x}^{2}, \bar{\xi}=\left(\xi^{+}+\xi^{-}\right) / 2$,

$$
\xi^{ \pm}=\frac{\tilde{\omega}+\tilde{k}_{x} \tilde{U}_{x}^{+}-\tilde{k}_{y} \tilde{U}_{d} \pm n \tilde{\omega}_{B}}{\sqrt{2} \tilde{k}_{z}},
$$

and $Z(\sigma)=\sqrt{\pi} \int \exp (-\tau)(\tau-\sigma)^{-1} d \tau$ is the plasma dispersion relation assuming a Maxwellian distribution function for electrons. In the limit of $k_{z} \rightarrow 0$, the 3D dispersion relation reduces to its $2 \mathrm{D}$ version, i.e., Eqs. (1) and (2).

It is known that the resonance peaks of the cyclotron motion, which are present in the $2 \mathrm{D}$ dispersion, become 
smoothed in the presence of a nonzero $k_{z} \lambda_{D}$, leading to a broadband ion acoustic-like spectrum. It is important to note that the 3D ECDI is different from an ion-acoustic instability that is derived assuming nonmagnetized electrons. As the 3D spectra result in a broadband (nonresonant) solution $[19,32]$, the growth rates of the ECDI can become comparable to those of the IITSI and the demarcation between the different modes which is evident in Fig. 2(c) would be less clear. Comparison of a full 3D simulation and the 3D linear kinetic theory is reserved for future investigation.

\section{CONCLUSIONS}

This paper presents insights into the cross-field electron transport in partially magnetized plasmas due to the presence of multiply charged ions. Using a multidimensional kinetic simulation accounting for both singly and doubly charged ions, the nonlinear interaction between the ion-ion two-stream instability (IITSI) and electron cyclotron drift instability (ECDI) is investigated. The present study discusses the effects of IITSI driven by the multiply charged ion streams on electron and ion transport, while fixing the plasma characteristics, which sets up the ECDI.

While it has been considered that the azimuthal plasma wave (in the direction of $E \times B$ drift) may be the dominant contributor to turbulent electron transport across the magnetic field, the present paper illustrates that the plasma wave excited in the axial direction (parallel to the applied electric field) and its coupling with the azimuthal ECDI further enhances cross-field diffusion. Numerical simulations presented in this work reveal the presence of the IITSI driven by the relative velocity between accelerated ions of different charge states $\left(\mathrm{Xe}^{+}\right.$and $\mathrm{Xe}^{2+}$ in the present study). This mode, coupled to the ECDI via the $E \times B$ drift of electrons, was first detected using coherent Thomson scattering measurements and an analytical basis for its appearance was proposed in Ref. [20]. The simulation results presented in this paper capture the features of the instability studied experimentally and analytically and it is observed that the coupling of the ECDI and IITSI enhances the cross-field electron transport by almost $90 \%$ of the contribution due to ECDI alone. Although the linear kinetic theory predicts a growth rate for the IITSI which is smaller than that of the ECDI, the nonlinear saturation (and, in particular, the nonlinear coupling) of the various instabilities plays an important role in the electron transport across the magnetic field.

The plasma wave excited in the axial direction also leads to the broadening of the ion velocity distribution functions. Since the phase velocity of the plasma wave lies between the velocities of the singly and doubly charged ion streams, the trapping of doubly charged ions occurs even with smallamplitude plasma waves in the axial direction. As the doubly charged ion fraction increases, the amplitude of the plasma wave driven by the IITSI increases and both the singly and doubly charged ions become trapped by the axial plasma wave. This leads to decrease in the bulk velocity of $\mathrm{Xe}^{2+}$ and broadening of the $\mathrm{Xe}^{+}$ion VDF.

\section{ACKNOWLEDGMENTS}

This material is based on work supported by the Air Force Office of Scientific Research under Award No. FA9550-181-0090 and by the US Department of Energy, Office of Science, Office of Fusion Energy Sciences, under Award No. DE-SC0020623. The authors acknowledge the Texas A\&M High Performance Research Computing Center. The authors acknowledge D. Grésillon, C. Honoré, A. Héron, N. Lemoine, and I. D. Kaganovich for prior discussions and the referees for their valuable feedback on the manuscript.
[1] J.-P. Boeuf, J. Appl. Phys. 121, 011101 (2017).

[2] K. Hara, Plasma Sources Sci. Technol. 28, 044001 (2019).

[3] S. Yoshikawa and D. J. Rose, Phys. Fluids 5, 334 (1962).

[4] D. W. Forslund, R. L. Morse, and C. W. Nielson, Phys. Rev. Lett. 25, 1266 (1970).

[5] D. W. Forslund, R. L. Morse, and C. W. Nielson, Phys. Rev. Lett. 27, 1424 (1971).

[6] M. Lampe, W. M. Manheimer, J. B. McBride, J. H. Orens, R. Shanny, and R. N. Sudan, Phys. Rev. Lett. 26, 1221 (1971).

[7] S. P. Gary and J. J. Sanderson, J. Plasma Phys. 4, 739 (1970).

[8] L. Muschietti and B. Lembège, Adv. Space Res. 37, 483 (2006).

[9] J. McBride, E. Ott, J. Boris, and J. Orens, Phys. Fluids 15, 2367 (1972).

[10] S. Matsukiyo and M. Scholer, J. Geophys. Res. 108, 1459 (2003).

[11] E. R. Priest and J. J. Sanderson, Plasma Phys. 14, 951 (1972).

[12] C. T. Dum, Phys. Fluids 21, 945 (1978).

[13] K. Hara and C. Treece, Plasma Sources Sci. Technol. 28, 055013 (2019).

[14] L. Muschietti and B. Lembège, J. Geophys. Res. Space Phys. 118, 2267 (2013).
[15] J.-C. Adam, A. Héron, and G. Laval, Phys. Plasmas 11, 295 (2004).

[16] A. Ducrocq, J.-C. Adam, A. Héron, and G. Laval, Phys. Plasmas 13, 102111 (2006).

[17] A. Héron and J.-C. Adam, Phys. Plasmas 20, 082313 (2013).

[18] S. Tsikata, N. Lemoine, V. Pisarev, and D. Grésillon, Phys. Plasmas 16, 033506 (2009).

[19] J. Cavalier, N. Lemoine, G. Bonhomme, S. Tsikata, C. Honoré, and D. Grésillon, Phys. Plasmas 20, 082107 (2013).

[20] S. Tsikata, J. Cavalier, A. Héron, C. Honoré, N. Lemoine, D. Grésillon, and D. Coulette, Phys. Plasmas 21, 072116 (2014).

[21] S. Tsikata and T. Minea, Phys. Rev. Lett. 114, 185001 (2015).

[22] P. Coche and L. Garrigues, Phys. Plasmas 21, 023503 (2014).

[23] T. Lafleur, S. D. Baalrud, and P. Chabert, Phys. Plasmas 23, 053503 (2016).

[24] J.-P. Boeuf and L. Garrigues, Phys. Plasmas 25, 061204 (2018).

[25] S. Janhunen, A. Smolyakov, D. Sydorenko, M. Jimenez, I. D. Kaganovich, and Y. Raitses, Phys. Plasmas 25, 082308 (2018).

[26] F. Taccogna, P. Minelli, Z. Asadi, and G. Bogopolsky, Plasma Sources Sci. Technol. 28, 064002 (2019).

[27] S. P. Gary and N. Omidi, J. Plasma Phys. 37, 45 (1987). 
[28] C. L. Grabbe, Geophys. Res. Lett. 12, 483 (1985).

[29] J.-E. Wahlund, F. R. E. Forme, H. J. Opgenoorth, M. A. L. Persson, E. V. Mishin, and A. S. Volokitin, Geophys. Res. Lett. 19, 1919 (1992).

[30] Y. Nakamura and Y. Saitou, Plasma Phys. Controlled Fusion 45, 759 (2003).

[31] S. D. Baalrud, C. C. Hegna, and J. D. Callen, Phys. Rev. Lett. 103, 205002 (2009).

[32] S. Tsikata, Ph.D. thesis, Ecole Polytechnique, 2009.

[33] T. Charoy et al., Plasma Sources Sci. Technol. 28, 105010 (2019).

[34] L. Garrigues, I. D. Boyd, and J.-P. Boeuf, J. Propul. Power 17, 772 (2001).

[35] F. S. Gulczinski, III and A. D. Gallimore, J. Propul. Power 17, 418 (2001).

[36] R. E. Waltz, Phys. Fluids 25, 1269 (1982).

[37] P. C. Liewer, Nucl. Fusion 25, 543 (1985).
[38] O. Chapurin and A. Smolyakov, J. Appl. Phys. 119, 243306 (2016).

[39] N. Dorval et al., J. Appl. Phys. 91, 4811 (2002).

[40] J. Bareilles, G. J. M. Hagelaar, L. Garrigues, C. Boniface, J.-P. Boeuf, and N. Gascon, Phys. Plasmas 11, 3035 (2004).

[41] K. Hara and K. M. Hanquist, Plasma Sources Sci. Technol. 27, 065004 (2018).

[42] J. Bohlmark, M. Lattemann, J. T. Gudmundsson, A. P. Ehiasarian, Y. Aranda Gonzalvo, N. Brenning, and U. Helmersson, Thin Solid Films 515, 1522 (2006).

[43] I. D. Kaganovich, G. Shvets, E. A. Startsev, and R. C. Davidson, Phys. Plasmas 8, 4180 (2001).

[44] E. K. Tokluoglu, I. D. Kaganovich, J. A. Carlsson, K. Hara, and E. A. Startsev, Phys. Plasmas 25, 052122 (2018).

[45] R. Kawashima, K. Hara, and K. Komurasaki, Plasma Sources Sci. Technol. 27, 035010 (2018).

[46] A. Ducrocq, Ph.D. thesis, Ecole Polytechnique, 2006. 